\title{
MIGRACIÓN DE LOS PADRES Y EL RENDIMIENTO ACADÉMICO DEL ESTUDIANTE
}

\section{PARENTAL MIGRATION AND PERFORMANCE STUDENT ACADEMIC}

\section{Referencia del artículo}

Saquimux, N. (2020). Migración de los padres y el rendimiento académico del estudiante. Revista Científica del SEP. 3(1), 1-8.

DOI: https: / / doi.org/10.36958/sep.v3i01.25

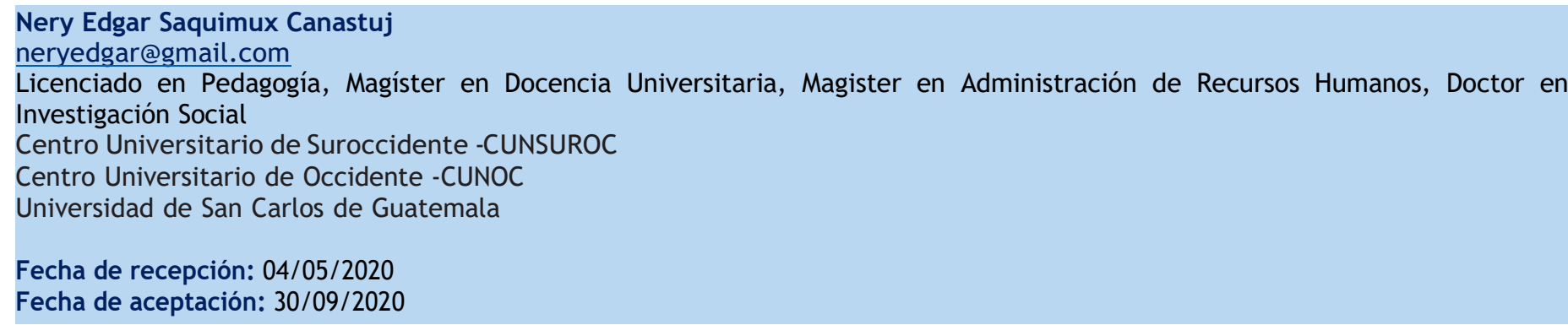

\section{RESUMEN}

La migración de padres de familia en búsqueda de trabajo en los Estados Unidos ha provocado el derrumbe del núcleo familiar con una secuela de efectos en la conducta de los hijos.

En esta investigación se abordó el impacto del abandono de los padres migrantes en la conducta escolar y rendimiento académico de los jóvenes de los institutos del nivel medio del departamento de Suchitepéquez. Fue un estudio de casos que abarcó en su primera parte la relación entre la migración paterna y el rendimiento académico de los jóvenes.

El efecto que resalta la investigación es la percepción de los jóvenes en que su futuro no está en la migración de su país sino en la salir adelante con sus estudios, tratar de culminar una carrera universitaria, hacer una familia y vivir en Guatemala.

A pesar de que la falta de la figura paterna afecta su disciplina en su desarrollo escolar, los jóvenes mostraron una prematura madurez psicológica que los ubica en su realidad y ven la vida como un compromiso personal de buscar el éxito preparándose académicamente y no migrar de Guatemala como lo hicieron sus padres.

\section{PALABRAS CLAVE}

Migración, rendimiento académico, autoestima, conucta escolar, frustración

\section{ABSTRACT}

The migration of parents in search of work in the United States has caused the collapse of the family nucleus with a sequel of effects on the behavior of their children.

In this research, the impact of the abandonment of migrant parents on the school behavior and academic performance of young people from high schools in the department of Suchitepéquez was addressed. It was a case study that covered in its first part the relationship between paternal migration and the academic performance of young people.

The effect highlighted by the research is the perception of young people that their future is not in the migration from their country but in getting ahead with their studies, trying to complete a university degree, start a family and live in Guatemala.

Despite the fact that the lack of the father figure affects their discipline in their school development, the young people showed a premature psychological maturity that places them in their reality and they see life as a personal commitment to seek success by preparing academically and not migrating from Guatemala like their parents did.

\section{KEYWORDS}

Migration, academic performance, self-esteem, school behavior, frustration 


\section{1.- INTRODUCCIÓN}

La inmigración ha sido una característica del ser humano a lo largo de su historia. Desde que apareció sobre la faz de la tierra, su tendencia natural ha sido inmigrar en búsqueda de mejores condiciones de vida.

El estadio nómada del desarrollo de la humanidad fue marcado por un modelo de vida basado en la inmigración de las hordas primitivas por todos los territorios del planeta que les ofreciera condiciones de supervivencia tales como: disponibilidad de alimentos, agua y medios de protección ante el embate de los fenómenos naturales. En ese entonces, la inmigración se dio implícita a la forma natural del ser humano de sobrevivir y conservar su especie.

El primer gran proceso de migración lo lleva a cabo el hombre primitivo. Las primeras migraciones prehistóricas sucedieron durante el período Paleolítico, las que supusieron la salida del Homo sapiens de África hace 200.000 años y su expansión por todo el planeta. Esta expansión se realiza desde África hacia Eurasia y se produce después de un gran cambio ambiental, pues tuvo lugar una acusada y prolongada sequía que pudo ser la razón de salida que motivara la migración. Las primeras migraciones humanas se dieron por la búsqueda de comida y nuevos territorios en los cuales esos individuos pudieran sobrevivir. Los pocos pobladores existentes se encontraban distribuidos en el continente africano, formando pequeños grupos familiares, dedicados a la caza, la pesca y la recolección. (Yanes, 2018, pág. 2)

La inmigración fomentó en los seres humanos su capacidad de adaptarse a diversos climas y esa experiencia le proveyó a su organismo condiciones físicas que afianzaron y fortalecieron su capacidad biológica para sobrevivir en diferentes regiones del planeta. La movilidad del ser humano propició su capacidad de adaptación a la naturaleza y a las diversas condiciones de sobrevivencia.

La humanidad se expande desde el principio de los tiempos, el hombre "nace" nómada en su cuna africana del Valle del Rift; luego el sedentarismo perfilará las diversas razas que retomarán la dinámica migratoria. Gracias al nomadismo se pobló el planeta durante milenios y con él, la humanidad sobrevivió y se adaptó a fenómenos naturales o territorios hostiles. (Sanfeliu, 2010, pág. 53)

Era de esperar que la expansión de las hordas nómadas propiciase automáticamente el intercambio de culturas y modos de vida entre los grupos de hombres primitivos en proceso de expansión. Las estirpes de éstos comenzaron a generar fuerzas de dominación entre sí, de tal manera que por "ley del más fuerte"; unos grupos dominaron a otros, sometiéndolos a procesos de conquista territorial, económica y cultural.

De esa cuenta, las hordas primitivas que sobrevivieron con mayor éxito a los embates de la naturaleza y de los depredadores, adquirieron más posibilidades de dominar a los demás grupos de humanos, especies animales y recursos naturales, bajo las condiciones de vida del hombre sedentario.

Al respecto se tiene que, en una pequeña zona convivieron neandertales, cromañones y, quizá, esos otros homínidos cuyo ancestro común dataría de un millón de años. El Homo erectus fue el primer nómada. Dejó África mucho antes de que se pusiera en marcha el Homo sapiens hace 50.000 años para reemplazar a otras especies. La masificación trae consigo las luchas que llevan al cromañón a afinar el ingenio y expandirse como Sapiens. Las viejas migraciones fuerzan la adaptación a nuevos escenarios y el intelecto ganó con ello; el humano que deja África lo hace ya con capacidad de abstracción, los intercambios genéticos así incorporados introducen mayor complejidad de pensamiento. (Sanfeliu, 2010, pág. 67)

Como se dijo anteriormente, la inmigración siempre tuvo motivos de sobrevivencia, pero también tuvo como factor de impulso la natural curiosidad humana de descubrir nuevos derroteros y encontrar nuevas respuestas a sus intenciones de desarrollo genético, económico y social. Con ello se logró dar mayor celeridad al proceso de hominización. "Hace más de dos millones de años la migración no fue concebida como una solución desesperada, sino como la necesidad de dar respuesta a la curiosidad humana y de superar nuevos retos relacionados con nuestra capacidad de adaptación" (Zanz, 2016 , pág. 11)

La actividad inmigratoria de los seres humanos resultó ser una de las propiedades sustantivas de su historia por la cual ha evolucionado poblando todas las regiones del planeta. En tal virtud se puede deducir que la movilidad de los seres humanos es una práctica inherente a su existencia como especie inteligente y ocurre sobreponiéndose a cualquier obstáculo.

Con el correr del tiempo, las poblaciones sedentarias diseminadas en el planeta, provocó el desarrollo general de las civilizaciones que construyeron su propio conjunto de costumbres, ideas, creencias, conocimientos científicos y técnicos, dando como resultado el surgimiento de la multiplicidad de culturas acordes a las condiciones climatológicas de donde se asentaron.

Las civilizaciones marcaron un momento de evolución de la humanidad en su constate empeño por dominar la naturaleza 
y separarse constantemente del reino animal. El proceso civilizatorio provocó que los seres humanos superaran en número de miembros y grupos étnicos al grado de que algunos de éstos con mejor desarrollo subyugaron a los grupos menos afortunados.

El concepto de civilización surge a lo largo del siglo XVIII e inicialmente se identificó con la forma de vida y los conocimientos de las sociedades avanzadas, contraponiéndose al término de barbarie que se atribuía a la forma de vida y los conocimientos de los pueblos colonizados o más atrasados. De este modo, los franceses, británicos y norteamericanos utilizarán el término civilización para referirse al conjunto de elementos espirituales que posee una sociedad, nacional o internacional, mientras que el término cultura lo aplicarán preferentemente a los aspectos espirituales que caracterizan la personalidad de los individuos, de ahí que cuando se analizan los fenómenos sociales se utilice el primero de ambos términos. (Calduch, 2003, pág. 3)

Una civilización es un constructo de convivencia humana más amplia, por lo que cuando se señala a un grupo de seres humanos como "civilizado", se hace referencia al agrupamiento de individuos cuya diversidad cultural permite ampliar su visión del mundo y con ello disponer de amplia capacidad de creatividad para dominar a otros grupos de mayor homogeneidad cultural.

La civilización se define por elementos objetivos comunes, tales como la lengua, historia, religión, costumbres, instituciones y por la autoidentificación subjetiva de la gente. (...) Las civilizaciones son el "nosotros" más grande dentro del que nos sentimos culturalmente en casa, en cuanto distintos de todos los demás “ellos” ajenos y externos a nosotros. (Grajel, 2018 , pág. 18)

En la cultura occidental, el término civilización se utiliza para referirse al conjunto de elementos espirituales que posee una sociedad, nacional o internacional, en el sentido de que éstos son inmateriales, pero se encuentran compartidos por una ligazón identitaria de los miembros. El término cultura se aplica preferentemente a los aspectos espirituales que caracterizan específicamente la personalidad de los individuos que conviven en un mismo contexto histórico y son recipiendarios de una cultura construida por las generaciones ancestrales, de tal manera que se reproduce "genéticamente" a los nuevos miembros de la sociedad al nacer y contactar con dicho contexto histórico.

En síntesis, el proceso de hominización se aceleró con el estilo de vida del hombre sedentario quien al asentarse en un solo lugar pudo organizar el trabajo de sus miembros y crear la infraestructura vital propia de una especie de seres vivos, inteligentes y racionales. Desde ese momento, los seres humanos tienden a preferir por vivir en mejores condiciones de vida y de subsistencia, por lo que habitantes de contextos menos favorecidos tiende a inmigrar a otros destinos que ofrecen mejores condiciones de vida y de subsistencia.

La inmigración implica en términos prácticos cruzar las fronteras que en la práctica se refiere a la conquista de nuevos territorios de manera individual o grupal para mejorar la calidad de vida.

El ánimo de moverse de un lugar a otro para conocer nuevas culturas es inherente al ser humano. por ello ha pervivido a lo largo de la historia, aunque cada sociedad le imprime sus propios matices. La migración es un fenómeno social total, para usar un concepto de Marcel Mauss; sus dimensiones y efectos son de tal magnitud y están entrelazados de tal manera que no es posible analizar cada elemento de manera aislada. Los motivos para migrar, la decisión, el trayecto, la inserción en la sociedad receptora y los vínculos con la sociedad de origen son distintas aristas de la experiencia. La migración siempre deja una huella indeleble en la vida de cada sujeto que la experimenta. (Yercko \& Falcón, 2012, pág. 301)

La sociedad se ha mantenido en constante movimiento. La movilidad de la sociedad en general obedece a su tendencia natural de intercambiar culturas puesto que a, toda movilidad humana la acompaña su cultura y puede encontrarse con otras, interactúa a cada paso, avanza progresivamente hacia contenidos derivados de sí o bien que surgen del diálogo y del encuentro con lo nuevo. La migración sin duda impacta en la cultura, al igual que la cultura forma parte de los factores de la migración y es su compañera de viaje en todo momento. (Guillen, 2016, pág. 15)

En el caso de los guatemaltecos que emigran hacia los Estados Unidos de Norteamérica y otros países ubicados en ese rumbo geográfico, obedece a la búsqueda de empleo con mejores salarios y por consiguiente asegurar la sobrevivencia económica de sus familias que se debaten en la pobreza y extrema pobreza en Guatemala.

La globalización tiene varias dimensiones (económica, política, jurídica, cultural) y cada una de ellas marca ciertas pautas para los procesos migratorios. La más visible es la económica. Los análisis desde esta perspectiva abordan los flujos de capital que se mueven cotidianamente, el peso que han adquirido las remesas como alivio a la pobreza y como factor de expulsión (Yercko \& Falcón, 2012, pág. 301) de un fuerte contingente de población económicamente activa de los países pobres, que tiene que emigrar para asegurar la sobrevivencia de sus familias. 
La pobreza de la población guatemalteca se caracteriza por el bajo nivel de calidad de vida. Ser pobre en Guatemala es vivir en casas precarias, estar vulnerable a padecer de desnutrición crónica, estar en peligro de morir por enfermedades prevenibles, ser analfabeta o bien tener un bajo nivel de escolaridad y ser explotado como mano de obra barata al desempeñar labores manuales o artesanales bajo condiciones de trato inhumano. En estas condiciones de sobrevivencia, el pobre está condenado a insertarse en el trabajo informal, sin prestaciones laborales y devengar bajos salarios que no alcanza para mantener económicamente a su familia que por lo general está integrada por más de 5 hijos.

Esta angustiante situación que viven los pobres de Guatemala estimula la inmigración ilegal de los padres de familia hacia los Estados Unidos en búsqueda de empleo. Tras su partida, los inmigrantes dejan en su país a su familia con hijos menores abandonándolos a su suerte en tan cruciales momentos de su vida. En Norteamérica les espera la persecución permanente del gobierno, puestos de trabajo manuales que no requiere créditos académicos, discriminación racial y sueldos que en cierta medida garantizan el sostenimiento mínimo de su familia que ha quedado desintegrada en Guatemala.

Ser migrante significa ser otro. Más específicamente, "el otro" o incluso "lo otro". Los extranjeros han sido definidos como peligrosos, lo cual es una definición amplia y generalmente ambigua. No se explica exactamente por qué son peligrosos, pero con la construcción de ese estigma se les despoja de su humanidad. Antes de ver a la persona, de reconocer una historia frecuentemente marcada por el dolor y la injusticia, se impone el rechazo. ... el Estado moderno se arroga la potestad de proteger a su población de esos peligros. Las políticas migratorias ilustran claramente este proceso. Los migrantes son clasificados, estigmatizados ante la opinión pública, amedrentados e invisibilizados. (Yercko \& Falcón, 2012, pág. 302) El ser inmigrante indocumentado en Estados Unidos tiene severas implicaciones al ser invisibilizados en el contexto de ese país. Esta situación los ubica en una paradoja, en el sentido de que, en el país receptor la sociedad no quiere reconocerlos, pero por su trabajo, ellos son necesarios para que los habitantes locales vivan su propio estilo de vida.

Los estigmas construidos sobre la población migrante son variados en su forma y sus alcances. En casos extremos se les considera pandilleros o delincuentes; de una manera más generalizada se les atribuyen características tales como la pereza, la suciedad, los malos modales, el desorden. Hugo S. Rojas explica la objetivación del rechazo: a simples verduras se les atribuyen características insalubres vinculadas con la nacionalidad y el precio se castiga. Ahí también se verifica la frontera cultural. Ser "otro" alude a la diferencia. Ser "el otro" implica una noción de alteridad: distinto, extraño, peligroso. Ser “lo otro" agrega la cosificación. (Yercko \& Falcón, 2012, pág. 304)

El gobierno de los Estados Unidos expulsa a los inmigrantes porque su estancia en ese país riñe con sus leyes de migración y los considera delincuentes que ponen en peligro la seguridad de sus ciudadanos. Por esa razón la permanencia ilegal de los inmigrantes en Estados Unidos los coloca en riesgo inminente de ser capturados y deportados a su país de origen.

\section{2- MATERIALES Y MÉTODOS}

La investigación se desarrolló con un enfoque cualitativo aplicando la técnica de estudio de casos, los cuales fueron seleccionados intencionalmente dentro de la población de estudiantes del nivel medio del departamento de Suchitepéquez, en el suroccidente de Guatemala. El objetivo de este estudio fue identificar los efectos de la inmigración de los padres en el desempeño académico y el proyecto de vida de los jóvenes.

El supuesto de la presente investigación indicó que el tener uso de razón, al momento en que los padres decidieron migrar a los Estados Unidos, determina el rendimiento académico de los hijos en el nivel primario y medio de educación, debido a que los efectos del abandono paternal se viven con más contundencia cuando el niño o joven está consciente de la realidad, de que ello implica su abandono paternal. Este efecto es menor cuando los padres han migrado dejando a sus hijos menores de siete años.

El criterio para seleccionar los casos de estudiantes cuyos padres migraron a los Estados Unidos fue conformar dos grupos de estudio:

- Grupo A, jóvenes cuyos padres migraron cuando ellos tenían entre 0 y 7 años.

- Grupo B, los jóvenes cuyos padres migraron cuando ellos tenían entre 8 a 15 años.

- La evaluación del impacto de la inmigración de los padres en el rendimiento académico de los hijos se analizó tomando en cuenta el nivel de uso de razón de los jóvenes al momento en que sus padres tomaron la decisión de migrar y abandonar el seno familiar. 
Se contactaron a seis centros educativos del nivel medio del departamento de Suchitepéquez, en los cuales se estudiaron trece casos de estudiantes, siete mujeres y seis varones. Para recabar la información se solicitó a los estudiantes su historia de vida y se les aplicó dos entrevistas a profundidad que recopilaron sus impresiones en torno a su sentir personal respecto a la falta de su padre o madre, o ambos en el seno del hogar durante sus años de infancia y adolescencia y; como ese abandono ha influido en su rendimiento académico en el nivel primario y medio de educación.

Las entrevistas se aplicaron en dos instancias de réplica, en la primera aproximación se ahondó en los eventos de "recuerdo de impacto" narrados en sus respectivas historias de vida y en la segunda aproximación, se profundizó en su sentir actual y su rendimiento académico tanto en el nivel primario como en el nivel medio de educación.

Estas versiones fueron corroboradas y contrastadas con las opiniones, que, sobre el comportamiento escolar y rendimiento académico de los estudiantes vertieron los profesores y directores de los establecimientos educativos donde actualmente estudian. De esta manera se logró la triangulación de la información.

\section{3.- RESULTADOS}

La idea de buscar empleo y mejores condiciones de vida en territorio norteamericano lleva a los padres de familia a inmigrar ilegalmente a dicho destino, dejando en su hogar a sus hijos (niños y adolescentes) sin tutoría y orientación para enfrentar la vida, de tal manera que ellos crecen sin mayor protección tanto afectiva como moral, por lo que su formación y educación escolarizada presenta el faltante de la influencia paternal en el hogar para generar su educación integral.

La educación de los hijos de los padres migrantes se torna problemática y con grandes riesgos de ser frustrada. Un artículo de prensa, citando declaraciones de la ex ministra de educación María del Carmen Aceña, destaca que:

No se descarta que la migración conlleve a que más jóvenes dejen la escuela, pues la situación económica y la falta de oportunidades, principalmente en las zonas rurales, los empuja desplazarse a otro país, principalmente Estados Unidos. Aunque no solo se trata de migración internacional, la interna también juega un papel importante, .... principalmente en aquellos hogares cuya economía depende de alguna actividad agrícola. (Ola, 2019, pág. 6)

El problema se agiganta, cuando los niños y jóvenes en edad escolar, tienden a emigrar ilegalmente a Norteamérica con la idea de encontrarse con sus padres e intentar reconstruir en tierra extranjera el núcleo familiar. La nota de Prensa Libre expone que, en el año 2018, la Organización Internacional para las Migraciones (OIM) reportó que un total de 4 mil 821 menores no acompañados retornaron a Guatemala luego de intentar viajar de manera ilegal hacia Estados Unidos en busca de mejorar sus condiciones de vida. La migración se convierte en un factor que repercute en la deserción escolar, pues los menores dejan la escuela para emprender ese viaje, si logran llegar a su destino ya no vuelven a su escuela, pero si no corren con esa suerte, es probableque tampoco lo hagan, y la mayoría vuelve a intentar cruzar la frontera. (Ola, 2019, pág. 6)

Esta nota periodística señala uno de los efectos del fenómeno migratorio hacia Norteamérica en la vida escolar de los niños y jóvenes. Sin embargo, otro efecto local que provoca la inmigración se vive en el escenario de los institutos del nivel medio, donde los jóvenes que fueron abandonados cuando tenían de ocho a más años manifestando indisciplina en su desempeño estudiantil, el cual es irregular, son rebeldes ante el cumplimiento de las normas institucionales y por consiguiente, su rendimiento académico es bajo.

En efecto, el director del Instituto por Cooperativa de San Pablo Jocopilas haciendo referencia a los efectos de la situación de abandono de los jóvenes por parte de sus padres migrantes, refirió el caso de "Homero" originario del municipio de Samayac, cuyo padre migró cuando él tenía 2 años; quien manifiesta en el instituto el comportamiento de un "joven de carácter fuerte, que ha mostrado falta de compromiso con sus responsabilidades de estudiante. Poco a poco ha cambiado al llegar a su adolescencia; la no presencia del papá le ha posado factura. La migración afecta al joven en su formación de valores, las buenas costumbres, tener una visión de vida clara y apartarse de las malas amistades para no confundir la libertad con libertinaje. (Mayorga, 10.9.19. 14:00 hrs.) “

A este respecto, la directora de un instituto de educación básica por cooperativa del municipio de Chicacao opinó que en el caso del estudiante: "Nogal" originario del municipio de Santa Catarina Ixtahuacán del departamento de Sololá, "Le ha afectado la migración de sus padres en cuanto a que es desinteresado en sus estudios, no asiste a la escuela, y su estado de ánimo demuestra tristeza y desesperación" ("Sánchez, 4. 9. 2019. 16:20 hrs.)

Por su lado, el director del Instituto por Cooperativa de San Pablo Jocopilas dijo en la entrevista que los jóvenes cuyos padres están ausentes por haber migrado a otro país, suelen mostrar un comportamiento de rebeldía y agresividad en el 
entorno escolar. Adujo el entrevistado que en caso del joven "Otoño" le ha, ...afectado el hecho de estar separado del padre y la mamá, puesto que al llegar a la adolescencia dicha situación ha representado episodios de agresividad y él ve en el ambiente de su establecimiento educativo un lugar para descargar su frustración al respecto. Es agresivo ya que la mamá transmite eso y el papá es irresponsable porque no les brinda lo necesario (Mayorga, 10.9.19. 14:00 hrs.),

\section{... debido a que se encuentra ausente del hogar}

A nivel de la organización de los institutos nacionales de educación básica y diversificada que operan en el departamento de Suchitepéquez, la atención psicopedagógica a los jóvenes adolescentes es mínima, dado a que la mayoría de éstos no poseen oficinas o Departamentos de Orientación Educativa; por esa razón los jóvenes con bajo rendimiento académico y comportamiento irregular no son atendidos técnicamente sino suelen ser sancionados por su comportamiento conflictivo y su indisciplina estudiantil.

En el caso de "Lucesela" una joven del municipio de Cuyotenango, que fue abandonada por su padre migrante cuando tenía 2 años, ha provocado su bajo rendimiento académico. Al respecto dijo: “En mis estudios voy perdiendo un año del nivel primario. Siempre he salido raspadito casi nunca obtengo buenas notas" ("Lucisela", 4.9.19. 13:30 hrs.) En esa misma dirección "Selva" una señorita de 17 años originaria de Samayac indicó: "En primaria fueron dos veces que repetí mi grado. ("Selva", 5.9.19. 14:30 hrs.) lo mismo le ocurrió a "Aurora" una joven proveniente del departamento de Sololá que fue abandonada por su padre migrante a los 13 años; quien afirmó haber repetido un grado del nivel primario y que actualmente su promedio es de 57 puntos.

Por su lado, "Primavera" originaria de Chicacao quien experimentó el abandono paterno por la migración cuando tenía 8 años, informó haber repetido el primer grado de educación básica, dijo que su rendimiento académico es regular y que actualmente está repitiendo el tercero básico.

Otros testimonios al respecto lo presentaron "Cereza" de Chicacao, que vio la partida de su padre a la edad de 15 años, ella indicó en la entrevista: “En mis estudios voy más o menos. Perdí un poco de interés en mis estudios y en la entrega de tareas, desde que mi padre partió a los Estados Unidos para trabajar" ("Cereza", 17.9.19. 17:30 hrs. ) De igual manera se expresó “Nogal” un joven de Santa Catarina Ixtlahuacán Sololá quien no ve a su padre desde la edad de 3 años y que al igual que los jóvenes anteriores indicó que ha repetido el segundo grado de educación primaria y actualmente tiene un promedio de 56 puntos.
Como se puede ver los testimonios anteriores indican que el bajo rendimiento académico es el denominador común de estos casos y que el efecto de la inmigración de los padres de familia afecta el rendimiento académico de sus hijos.

Cabe resaltar que las estudiantes mujeres cuyos padres emigraron cuando ellas no habían nacido o tenían de 2 o 3 años, en sus relatos revelaron que sus expectativas de vida académica son halagüeñas puesto que, en el caso de la joven "Bengala", del Instituto de San Lorenzo, cuyo padre emigró cuando ella tenía 2 años; dijo, pensando en su futuro: “Pienso estudiar magisterio para ayudar a los niños, me gusta convivir con ellos, es algo bonito. Yo voy bien en mis estudios. No he repetido ningún grado solo he obtenido punteos de 90 puntos. El más bajo fue 65" ("Bengala”, 10.9.2019 14:30 hrs. )

Ella es una excepción a la regla al igual que "Alba" de Cuyotenango, quien no había nacido cuando su padre emigró y al hablar de su rendimiento académico informó que no ha reprobado ningún grado y en el futuro desea estudiar y alcanzar una licenciatura.

En esa misma perspectiva de futuro académico se ubicó lo declarado por "Lucesela” de San Lorenzo, quien dijo “Pienso seguir estudiando en la universidad Criminología forense y trabajar tal vez más adelante, formar una familia, pero todo depende de Dios" (“Lucesela", 13.9.19. 16:00 hrs.) La expresión final de "Lucesela", denota un sentimiento de abandono frente a su futuro, al grado de dejarlo todo en manos de Dios. El sentirse sola ante el mundo sin el respaldo inmediato de su padre la lleva a pensar que el éxito académico y de su vida personal depende únicamente de la voluntad de Dios ya que en su vida no ha tenido el soporte y guía que le pudo ofrecer la compañía permanente de su padre.

En cuanto a las estudiantes mujeres, cuyos padres migraron cuando ellas tenían más de ocho años, como son los casos de "Selva", oriunda de Samayac, quien vio migrar a su padre cuando ella tenía 17 años; "Cereza” de Chicacao que tenía 15 años cuando fue abandonada por su padre y "Primavera" que a los 8 años vio migrar a su padre; la investigación reveló que tal acontecimiento las hizo madurar prematuramente y pensar proactivamente por educarse y superarse académicamente.

Con ese mismo sentimiento de abandono y la búsqueda de un asidero de consuelo para enfrentar la vida, "Selva" coincidió con las anteriores expresiones, cuando indicó que:

En el ciclo básico gracias a Dios y el apoyo de mi mamá mis calificaciones para ella y para mi han sido excelentes. Quiero seguir estudiando para que en mi futuro sea alguien muy importante y .... quiero ser doctora o enfermera para ayudar a 
las personas. ("Selva", 14.9.19. 14:39 hrs.

Nuevamente aparece la visión de Dios como el último consuelo de esperanza, protección y ayuda para enfrentar su vida.

La misma expectativa de desarrollo académico refirieron "Cereza”, quien no ha repetido ningún grado y a futuro desea seguir estudiando para ayudar a su mamá, independizarse y tener un esposo e hijos y; "Primavera” quien indicó que a pesar de haber repetido el primero básico, piensa trabajar para seguir estudiando en la universidad, ya que este año se graduará de bachiller en computación y ve en su educación la solución más viable para enfrentar su vida a futuro.

En cuanto a las expectativas de desarrollo académico y de vida personal de los estudiantes varones investigados, como es el caso de los jóvenes: "Homero" de Samayac, "Nogal" de Santa Catarina Ixtahuacan, “Otoño" y "Verano" ambos de Chicacao; que se quedaron sin la presencia de su padre migrante a la edad de 2, 3, y 4 años respectivamente; su expectativa académica a futuro se torna optimista, al coincidir en que, al no contar con el apoyo cercano de su padre, están dispuestos a salir adelante con su empeño y esfuerzo propio.

Esto se evidenció en el significado de sus expresiones dadas a conocer en la entrevista a profundidad. En el caso de "Homero" desea tener un trabajo estable y darle todo a su mamá porque ella y su padre le ayudaron a salir adelante. "Nogal" por su lado indicó que ahora piensa ayudar a la gente. Motivarla para lograr sus sueños, así como le motivaron a él sus padres.

En esa misma línea de pensamiento “Otoño” indicó que ha pensado en seguir estudiando en la universidad, pero si le gustaría tener una familia y estar pendiente de sus hijos, presume no haber reprobado ningún grado hasta ahora. Finalmente "Verano" quien debido a que no ha repetido clases y no ha dejado retrasadas piensa seguir estudiando en la universidad. Vale la pena resaltar que estos dos jóvenes al sobreponerse de la situación de abandono de sus padres migrantes han tomado plena conciencia de afrontar con responsabilidad sus estudios, lo cual se demuestra con el hecho de tener buen rendimiento académico en el instituto.

Lo importante aquí es el hecho de que los jóvenes analizados quieren quedarse en Guatemala y hacer una vida normal como futuros padres de familia. Esa perspectiva es el factor que los hace rendir en la escuela, aprovechar sus estudios y asegurarse una vida feliz viviendo con su propia familia.

Estos jóvenes piensan diferente a lo que afirma un autor consultado, que considera que la migración es una decisión que toman con más frecuencia los varones, al afirmar que:
Es posible que la movilidad femenina hacia Estados Unidos ocurra con menor frecuencia que la de los varones y la de otros desplazamientos femeninos dentro del país, porque las mujeres presentan una mayor permanencia en el lugar de origen o en el de destino y una periodicidad más relacionada con el ciclo de vida femenino, los roles de género y los riesgos que implica cruzar la frontera sin contar con documentos para trabajar o para internarse legalmente, y en menor medida asociada a las demandas del mercado laboral. (Aunguiano, 1998, pág. 71)

\section{4.- DISCUSIÓN}

El supuesto de la presente investigación indicó que el tener uso de razón al momento en que los padres decidieron migrar a los Estados Unidos, determina el rendimiento académico de los jóvenes en el nivel medio de educación, debido a que los efectos del abandono paternal se viven con más contundencia cuando el niño o joven está consciente que ello implica su abandono paternal. Este efecto es menor cuando los padres han migrado dejando a sus hijos menores de siete años.

El estudio puso al descubierto que la migración de los padres afecta emocionalmente a los jóvenes al grado de incidir en su autoestima, tener sentimientos de frustración y abandono, principalmente si ya tenían uso de la razón cuando sus padres decidieron emigrar. Esto se apreció en el 50\% de los casos analizados quienes reportaron tener un rendimiento académico deficitario.

Sin embargo, cabe resaltar que los estudiantes que fueron abandonados por sus padres migrantes cuando ellos aún no tenían pleno uso de la razón, desarrollan sus actividades académicas con responsabilidad y tienen en mente continuar sus estudios a nivel de la universidad, ejercer una profesión y realizarse como madres y padres de familia viviendo en Guatemala.

Lo anterior denota que el abandono de los niños en edades menores de 7 años por parte de los padres migrantes al final desarrolla en ellos una madurez emocional en forma prematura, puesto que, al no disponer de la cercanía física con sus padres, han asumido por si solos un comportamiento responsable en sus estudios y se formulan para su futuro perspectivas de vida basadas en su desarrollo académico y profesional.

Lo afirmado en el supuesto de la investigación confrontado a las versiones analizadas hasta aquí, demuestra que los hijos de inmigrantes que fueron abandonados por sus padres cuando ellos aún no hacían uso de la razón, presentan mejor rendimiento académico en el nivel medio, debido a que cuentan con mayor madurez emocional para enfrentar las responsabilidades de la vida y tienen la expectativa de realizar estudios universitarios, 
profesionalizarse y quedarse en Guatemala. En tanto que los jóvenes que vieron migrar a sus padres teniendo ellos ya uso de la razón; fueron impactados por dicho abandono lo cual les afectó emocionalmente por lo que su comportamiento en el ambiente escolar es irresponsable, conflictivo y por lo consiguiente su rendimiento académico es deficitario.

Por todo lo anterior se recomienda que en las escuelas e institutos del nivel medio se implementen Departamentos de Orientación que desarrollen programas de orientación juvenil para atender el impacto afectivo, emocional y de autoestima de los estudiantes cuyos padres de familia están ausentes por motivos de su inmigración hacia Norteamérica, esto con el fin de aprovechar su madurez psicológica prematura y ayudarlos a cumplir sus sueños de formarse profesionalmente cuando alcancen la edad adulta.

\section{5.- REFERENCIAS}

Aunguiano, M. (1998). Migración a la frontera norte de Méxicoy su relación con el mercado regional. Papeles de población, 63 - 79.

Calduch, R. (2003). Cultura y civilización en la sociedad internacional. Madrid, España: Universidad de San Pablo. CEU. .Grajel, F. (24 de febrero de 2018). La civilización mito. o realidad. La Razón, págs. 17 - 18.

Guillen, T. (2016). Migración y cultura. Tijuana, México: UNESCO Hernández, C., \& Poot, G. (2001). Conformación de valores en menores de edad en procesos de migración. Revista dePerinatología y Reproducción Humana, 69 - 74.

Ola, A. (31 de enero de 2019). Creciente migración obliga a los niños a abandonar las aulas. Prensa Libre, págs. 5 -6.
Oliva, L., León, D. , \& Rivera, E. (2007). ENSEÑANZA E INVESTIGACIÓN EN PSICOLOGÍA. Xalapa, México: CENIP. Sanfeliu, I (15 de marzo de 2010). Migración, racismo y poder Obtenido de https://www.imagoclinica.com/publicaciones/libros libros-psicología-psicoanalisis/migración-racismo-ypoder/

Yanes, B. (30 de noviembre de 2018). III Congreso Virtual Internacional Migración y Desarrollo. Obtenido de https://www. eumed.net/actas/18/migración/9-lasmigraciones-humanas- larga-peregrinacion-de-millonesde-anos.pdf

Yercko, N., \& Falcón, M. (2012). La migración y sus efectos en lacultura. Sociológica, 301 - 306.

Zanz, N. (2016). Migración y cultura. Tijuana México: UNESCO.

\section{Sobre autor}

\section{Nery Edgar Saquimux Canastuj}

Es Profesor del Área Curricular de Investigación de la carrera de Pedagogía del Centro Universitario de Suroccidente y de los Programas de Maestría del Centro Universitario de Occidente de la Universidad de San Carlos. Actualmente Coordinador del Programa de Doctorado Internacional de Desarrollo Territorial y Problemas Transfronterizos acreditado por la UNACH - CUNOC. Licenciado en Pedagogía, Magíster en Docencia Universitaria, Magister en Administración de Recursos Humanos, Doctor en Investigación Social con grado de PHD. Es conferencista nacional e internacional en temas de investigación. Dos publicaciones en revistas indexadas. Cuatro libros de texto sobre metodología de la investigación. Línea de investigación: Social cualitativa.

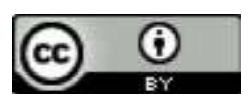

Este texto está protegido por una licencia Creative Commons 4.0.

Usted es libre para Compartir -copiar y redistribuir el material en cualquier medio o formato - y Adaptar eldocumento - remezclar, transformar y crear a partir del material- para cualquier propósito, incluso comercialmente, siempre que cumpla la condición de:

Atribución: Usted debe reconocer el crédito de una obra de manera adecuada, proporcionar un enlace a la licencia,e indicar si se han realizado cambios. Puede hacerlo en cualquier forma razonable, pero no de forma tal que sugieraque tiene el apoyo del licenciante o lo recibe por el uso que hace.

$\underline{\text { Resumen de licencia }}$ - Texto completo de la licencia 